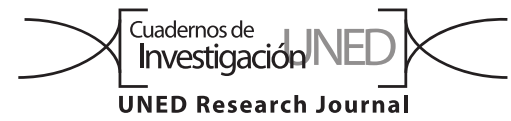

\title{
Comparación de dos métodos cromatográficos para determinar clorotalonil en un fungicida comercial
}

\author{
Esteban Pérez López ${ }^{1}$ \& Rosa Angélica Rodríguez Rodríguez ${ }^{1}$ \\ 1. Universidad de Costa Rica, Recinto de Grecia, Costa Rica; \\ esteban.perezlopez@ucr.ac.cr, (iD https://orcid.org/0000-0002-6379-7139, \\ rosdriguez@hotmail.com, (D) https://orcid.org/0000-0001-5304-8057 \\ Recibido 01-III-2019 • Corregido 28-V-2019 • Aceptado 06-VI-2019 \\ DOI: https://doi.org/10.22458/urj.v11i3.2627
}

\begin{abstract}
Comparison between two chromatographic methods to determine chlorothalonil in a commercial fungicide". Introduction: Chlorothalonil can be determined by several analytical techniques, including gas chromatography (GC) and high performance liquid chromatography (HPLC). These two techniques are used often because they have a higher level of stability and sensitivity. Objective: To compare the efficacy of analytical methodologies by GC and by HPLC for the determination of chlorothalonil in a commercial fungicide. Methods: Both the HPLC method and the GC method used by the laboratory were applied to equivalent samples and the results were compared with paired statistical tests. Results: Both methods produced statistically equivalent and reliable results. These parameters were satisfactory: selectivity, range of work and linearity, limit of detection and quantification, repeatability, intermediate precision, robustness, veracity and bias; we consider them linear, precise and exact methods that meet the norms of the Costa Rican Accreditation Entity (ECA). Conclusions: Both methods for determining chlorothalonil can be used reliably under the defined conditions.
\end{abstract}

Key words: Agrochemicals, chlorothalonil, pesticides, analytical method, validation.
RESUMEN: Introducción: El clorotalonil se puede determinar por varias técnicas de análisis, entre ellas la cromatografía de gases (GC) y cromatografía líquida de alta resolución (HPLC). Estas dos técnicas son las de mayor uso en los laboratorios Objetivo: Comparar la eficacia de las metodologías analíticas por GC y por HPLC para la determinación de clorotalonil en un fungicida comercial. Métodos: Aplicamos ambos métodos a muestras equivalentes y comparamos los resultados con pruebas estadísticas. Resultados: Ambos métodos arrojaron resultados estadísticamente equivalentes y confiables. Estos parámetros fueron satisfactorios: selectividad, ámbito de trabajo y linealidad, límite de detección y cuantificación, repetibilidad, precisión intermedia, robustez, veracidad y sesgo; los consideramos lineales, precisos y exactos que cumplen con las normas del Ente Costarricense de Acreditación (ECA). Conclusiones: Ambos métodos para determinación de clorotalonil se pueden utilizar de manera confiable bajo las condiciones definidas.

Palabras clave: Agroquímicos, clorotalonil, plaguicidas, método analítico, validación.
En el área de la agricultura se dan muchos problemas relacionado con plagas, las cuales hacen referencia a cualquier organismo vivo que compita u ocasione daños a las plantas o a sus productos y que puedan considerarse como tal, debido a su carácter económico, invasor o extensivo (Reyes, Vásquez, \& González, 2010). Los plaguicidas son sustancias o mezcla de sustancias utilizadas con la finalidad de prevenir, destruir, repelar o controlar cualquier plaga. Estas plagas pueden ser tan diversas como insectos, animales, hierbas, hongos y virus (Menéndez, 2009).

Los plaguicidas pueden ser naturales o sintéticos y una de las preocupaciones respecto a su uso es el riesgo de contaminación del agua y el suelo por las sustancias químicas utilizadas; especialmente porque debido a sus estructuras normalmente complejas, es difícil su degradación. En nuestros campos agrícolas se utiliza gran cantidad de fungicidas puesto que los hongos son plagas importantes dadas las condiciones climáticas propias de la región (Varés, 2006; Ministerio de Salud, 2007; Mendizábal, 2011).

Los plaguicidas fueron desarrollados para lograr un mejor crecimiento de la flora; gracias a su efectividad y bajo costo, permiten proteger los cultivos de casi cualquier amenaza. Sin embargo, a largo plazo se demostró que el uso indiscriminado de plaguicidas puede afectar 
al medioambiente e incluso puede modificar a las plagas, haciéndolas aún más resistentes a su uso (Álvarez, Conci, \& Peccoud, 2009; Ascuasati, 2012). Es indispensable que los plaguicidas solamente se usen si hay necesidad económica importante y de acuerdo con las recomendaciones de la etiqueta.

Los controles de plagas no intentan eliminar todas las plagas, ya que esto descompensaría el equilibrio natural de vida en el huerto. No todos los insectos son enemigos de los cultivos. Algunos insectos son polinizadores, otros ayudan a descomponer la materia orgánica y otros se alimentan de los insectos dañinos, con lo que a veces llegan a erradicar el problema sin que tengamos que intervenir. Es muy importante aprender a reconocer la plaga y/o el daño que causa para poder controlarla. En algunos casos son tan pequeñas que nuestra mejor herramienta de diagnóstico es el daño causado a las plantas (EcoTenda, 2013).

En la agricultura de nuestro país son frecuentes e importantes los daños que causan las plagas en los cultivos. Numerosas enfermedades se dan por medio de insectos, hongos y bacterias, que afectan tanto la salud de las plantaciones como la salud del ser humano y animales (Alatorre, 1999).

Las industrias de agroquímicos se centran en la búsqueda de productos menos nocivos para el ambiente y formulaciones más seguras para los usuarios y el entorno. Estas tendencias responden en buena medida a las exigencias de las agencias reguladoras de los países desarrollados para el registro y autorización de uso de los plaguicidas. Desde las primeras etapas en el desarrollo de plaguicidas, la industria toma en cuenta las disposiciones vigentes para su registro y aprobación (Decreto No 24337-MAG-S, 1995). De hecho, plaguicidas que pueden tener una excelente actividad, selectividad y nicho en el mercado, pueden ser desechados desde etapas iniciales del desarrollo si se prevé que no van a cumplir con los requisitos impuestos para el registro. (Villegas, 1993; Ferreira, 1996).

Existen varios tipos de plaguicidas, en este caso un fungicida es el objeto de estudio analítico. Los fungicidas son sustancias tóxicas que se emplean para impedir el crecimiento o para matar los hongos y mohos perjudiciales para las plantas, los animales o el hombre. Existen dos tipos de fungicidas, los de contacto y los sistemáticos (Pérez \& Forbes, 2017). Los fungicidas de contacto llamados también protectores, actúan solamente sobre la superficie de la planta donde el fungicida ha sido depositado y evitan que los esporangios germinen y penetren a las células. Por ello se recomienda cubrir la mayor parte de la planta con este tipo de productos. Los fungicidas sistémicos son absorbidos a través del follaje o de las raíces y se movilizan a toda la planta. Otros productos sistémicos, conocidos como fungicidas translaminares tienen la capacidad de moverse del lado superior de la hoja al inferior, pero no de hoja a hoja. Los fungicidas sistémicos afectan varias etapas de la vida del hongo (Pérez \& Forbes, 2017).

Específicamente, el fungicida implicado es el clorotaIonil, que proviene de la familia de los clorofenilos, los cuales se fijan a grupos sulfhidrilo y mercapto, es un fungicida de contacto, con un amplio espectro, similar a los ditiocarbamatos, y de numerosos efectos tóxicos en mamíferos (aumento de mutaciones, toxicidad crónica, reacciones alérgicas, probablemente cancerígeno). Estos productos no deben usarse con humectante ya que se disminuye la fungitoxicidad y aumenta la fitotoxicidad y son incompatibles con aceites o formulaciones oleosas. (Gepp \& Mondino, 2018).

La seguridad de que un producto cumple con las especificaciones, solo es relevante si los métodos a través de los cuales se llega a los resultados, son oficiales o están validados. De aquí la importancia de contar con procedimientos validados, lo cual según Duran (2011), se define como la acción en la que se prueba que cualquier material, proceso, procedimiento, actividad, equipo o mecanismo empleado en la fabricación o control, logrará los resultados para los cuales se destina. La calificación de los instrumentos utilizados en los procesos productivos, así como en la caracterización de los productos y su control de calidad, constituye un requisito previo a la validación de cualquier técnica analítica que haga uso de estos. (Álvarez, Támbara, Álvarez, Besada, \& González, 2014).

De acuerdo con estudios, el clorotalonil se puede determinar por varias técnicas de análisis, entre las cuales dos de ellas son: por cromatografía de gases (GC) y por cromatografía líquida de alta resolución (HPLC) (Miranda \& Martín, 2018). Estas dos técnicas son las de mayor uso en los laboratorios debido a que tienen un nivel más elevado de estabilidad y sensibilidad para detectar las sustancias deseadas, sin embargo, la mayoría de métodos encontrados son para residuos de los fungicidas en los productos y lo que se pretende determinar es el activo del producto terminado.

\section{MATERIALES Y MÉTODOS}

El método con el que trabaja el laboratorio de la empresa Formuquisa es el de cromatografía de gases, cuyo método está basado en el método oficial (AOAC, 2016) como referencia, sin embargo, se modificó a beneficio propio cambiando el solvente de metanol a 
acetona, manteniendo las demás condiciones iguales al método oficial.

Se planteó obtener otro método analítico por HPLC que brinde resultados confiables y seguros, como lo ha hecho el método de análisis por GC, esto con el fin de asegurar un nuevo método alterno que favorezca la operación de la industria en el aseguramiento de la calidad de sus productos y, en este caso, con HPLC que presenta ventajas analíticas y de costos en comparación con GC. La principal ventaja analítica es la mayor sensibilidad del método.

Los resultados del contenido de clorotalonil en un plaguicida deben cumplir con lo establecido (Cuadro 1) en la normativa vigente (Norma RTCR-171, 1991), que define las tolerancias permitidas para la concentración de ingrediente activo. Esta norma es basada, a su vez, en las especificaciones de la FAO (Organización de las Naciones Unidas para la alimentación y la Agricultura) para productos destinados a la protección de las plantas.

Para el clorotalonil, se tiene que el contenido de ingrediente activo es $72 \%$, entonces de acuerdo con el Cuadro 1 este producto pertenece al porcentaje de $\geq 50$, por lo tanto cuenta con una tolerancia de $\pm 2,5$ lo cual significa que los resultados de los análisis se pueden encontrar dentro del rango 69,5-74,5\% de ingrediente activo y serán aceptados, tal como lo aplica el laboratorio implicado para sus resultados de análisis.

Para efectos de este estudio, se implementó el método de análisis de clorotalonil por HPLC (García \& Pérez, 2012), utilizando las siguientes condiciones de

CUADRO 1

Tolerancias permitidas para el ingrediente activo en plaguicidas

\begin{tabular}{ccc}
$\begin{array}{c}\text { Porcentaje ingrediente } \\
\text { activo declarado }\end{array}$ & $\begin{array}{c}\text { Límites aceptables } \\
\text { unidades }\end{array}$ & $\begin{array}{c}\text { Límites aceptables en porcentajes } \\
\text { sobre el contenido declarado }\end{array}$ \\
250 & \pm 2.5 & No aplicable \\
$10-50$ & No aplicable & \pm 5 \\
$2,5-10$ & No aplicable & \pm 6 \\
$0-2,5$ & No aplicable & \pm 10 \\
\hline
\end{tabular}

Fuente: Norma RTCR-171 (1991).

trabajo: acetonitrilo-agua (70:30) como fase móvil, columna $\mathrm{L}=250 \mathrm{~mm}, \mathrm{ID}=4,6 \mathrm{~mm}$, Supelcosil LC-18 5 $\mathrm{m}$, flujo de $1,4 \mathrm{~mL} / \mathrm{min}, 5 \mu \mathrm{L}$ de volumen de inyección y $231 \mathrm{~nm}$ de longitud de onda, según condiciones facilitadas por una marca comercial (Sigma-Aldrich, 2016).

Se realizaron pruebas para determinar el tiempo de retención, así como el volumen de inyección (1, 5 y 10 $\mathrm{\mu L})$ y luego se realizaron curvas de calibración con cinco niveles para establecer el ámbito de trabajo. Se ensayaron muestras reales del plaguicida en diferentes lotes. Estas pruebas se compararon con las mismas realizadas por GC como método referente, y así determinar si son aceptables.

Se procedió a la validación del método por cromatografía líquida de alta resolución, y del método por cromatografía de gases utilizado por la empresa con regularidad y derivado del método oficial (AOAC, 2016) pero modificando el diluyente utilizado. Se siguieron todos los lineamientos de la guía del Ente Costarricense de Acreditación (Ente Costarricense de Acreditación, 2015) para validar métodos de ensayo, y los criterios de aceptación fueron tomados del Reglamento de Validación de
Métodos Analíticos Requeridos para el Registro Sanitario ante el Ministerio de Salud. Los parámetros a determinar son los siguientes:

Selectividad (prueba de placebo): Se prepararon aproximadamente $100 \mathrm{~mL}$ con las cantidades especificadas de sustancias en el formato de la formulación, sin el activo. Con el placebo realizado se pesaron de forma independiente seis muestras con la misma cantidad y preparación que define el método.

Criterio de aceptación: El porcentaje representado por el placebo debe ser menor a 3\%.

Ámbito o intervalo de trabajo: Se determinó con los niveles más bajos y más altos de concentraciones demostrados que pueden ser determinados con la precisión y la exactitud requeridas para la matriz de estudio. Criterio de aceptación: el coeficiente de correlación de la curva obtenida debe ser mayor a 0,995. Para determinarlo se calcula un porcentaje de error, cuyos resultados deben 
encontrarse en el rango del porcentaje de tolerancia para este producto $( \pm 2.5 \%)$, se calcula de la siguiente forma:

$$
\begin{gathered}
\text { \% Error }=100 *(\mathrm{CnE}-\mathrm{CnD}) / \mathrm{CnE} \\
\text { CnE: Concentración esperada. } \\
\text { CnD: Concentración Determinada. }
\end{gathered}
$$

Linealidad: Se realizaron tres curvas de calibración con soluciones al $80 \%, 90 \%, 100 \%, 110 \%$ y $120 \%$ de la concentración del ingrediente sugerida por el método analítico. Se verifica mediante la obtención de coeficientes de correlación mayor o igual a 0,995.

Límite de detección: Se prepararon soluciones a concentraciones del analito, y se determina la mínima concentración detectable, aunque esta no sea cuantificable. El límite de detección se calcula con la siguiente fórmula:

$$
\begin{gathered}
\mathrm{LD}=\mathrm{X}+3 * \mathrm{~S} \\
\mathrm{X}=\text { Promedio del Blanco } \\
\mathrm{S}=\text { Desviación de la muestra enriquecida }
\end{gathered}
$$

Límite de cuantificación: Se prepararon soluciones a concentraciones del analito y se determina cual es la mínima concentración que puede ser cuantificada con precisión y exactitud aceptables. El límite de detección se calcula con la siguiente fórmula:

$$
\begin{gathered}
\text { LC }=10 * \text { Desviación mínima * la pendiente } \\
\text { de la curva de calibración }
\end{gathered}
$$

Repetibilidad (precisión): Se prepararon e inyectaron de forma independiente seis estándares al 90\%, $100 \%$ y $110 \%$ del valor de concentración del analito en la muestra, según la concentración definida en cada técnica. Criterio de aceptación: el coeficiente de variación debe ser no mayor al $2 \%$.

Precisión intermedia: (Con cambio de equipo en GC y cambio de analista en HPLC); para el método por GC se realizaron las determinaciones en dos equipos de GC disponibles en el laboratorio, uno marca Agilent y otro marca Shimadzu. Para el método HPLC, se realizó cambio de analista. Para estas pruebas y la de repetibilidad se realizó una prueba del contraste $\mathrm{F}$ para la comparación de desviaciones estándar. En donde se plantea una hipótesis para probar que no haya diferencia significativa en las varianzas de los resultados y determinar cuál es más preciso. Para esto, se calcula el valor de F y se busca el valor teórico de F.

Robustez: Se prepararon ocho muestras independientes con concentraciones que coincidan con la curva de calibración. Se hicieron mediciones de las mismas muestras dos días después y se determinó el valor de variación. Criterio de aceptación: el porcentaje de variación no mayor $\mathrm{a}+3 \%$.

Exactitud: Para la evaluación de este parámetro se realizó el análisis de un material de referencia certificado, se prepararon muestras en tres niveles de concentración y se realizaron tres réplicas de cada muestra. La valoración de la exactitud se efectuó mediante el porcentaje de recuperación del analito, se compara el promedio de los valores obtenidos $(X)$ con el valor de referencia certificado $(m)$, teniendo en cuenta la incertidumbre asociada a ese material. El módulo de la diferencia da como resultado el sesgo del método: $\mathrm{s}=\mathrm{X}-\mathrm{m}$. Criterio de aceptación según la FDA para un producto terminado debe ser mayor a $97 \%$ y menor a $103 \%$.

También se evaluó la veracidad del método empleando cinco niveles de enriquecimiento, en los cuales, la recuperación también debe estar entre $97 \%$ y $103 \%$.

Al finalizar la validación de los métodos, se realizó una comparación entre los métodos por cromatografía de gases y por cromatografía líquida, en donde se prepararon y cuantificaron muestras reales del plaguicida en estudio, según se describe en el Cuadro 2.

CUADRO 2

Condiciones de trabajo para las muestras de clorotalonil según métodos por GC y HPLC

\begin{tabular}{lll}
\multicolumn{1}{c}{ Parámetro } & \multicolumn{1}{c}{ Método GC } & \multicolumn{1}{c}{ Método HPLC } \\
Diluyente & Acetona & Acetonitrilo \\
Peso de Muestra & $0,350 \mathrm{~g}$ & $0,07-0,09 \mathrm{~g}$ \\
Dilución & $50 \mathrm{~mL}$ & $50 \mathrm{~mL}$ y $5 \mathrm{~mL}$ en $50 \mathrm{~mL}$ \\
\hline
\end{tabular}

Ética, conflicto de intereses y declaración de financiamiento: Los autores declaran haber cumplido con todos los requisitos éticos y legales pertinentes, tanto durante el estudio como en el manuscrito; que no hay conflictos de interés de ningún tipo, y que todas las fuentes financieras se detallan plena y claramente en la sección de agradecimientos. Asimismo, están de acuerdo con la versión editada final del documento. El respectivo 
documento legal firmado se encuentra en los archivos de la revista.

\section{ANÁLISIS}

Al haber sometido a prueba el método por HPLC, según los resultados reflejados en el Cuadro 3 y basado en las especificaciones evidenciadas en el Cuadro 1, los resultados de las concentraciones del clorotalonil obtenido para los tres lotes del producto comercial ensayado por HPLC, se encuentran dentro de lo establecido en la Norma RTCR-171 (1991) y los valores obtenidos en desviación estándar relativa (DSR\%) se encuentran muy por debajo del $2 \%$ considerado como aceptable para la determinación. Esto da indicios de que el método por HPLC arroja resultados favorables preliminarmente.

CUADRO 3

Resultados de prueba en muestras de tres lotes de clorotalonil por HPLC

\begin{tabular}{cccc} 
Muestra & $\begin{array}{c}\text { Lote 1 } \\
(\% \mid \mathrm{A} \pm 0,03)\end{array}$ & $\begin{array}{c}\text { Lote 2 } \\
(\% \mid \mathrm{A} \pm 0,03)\end{array}$ & $\begin{array}{c}\text { Lote 3 } \\
(\% \mid \mathrm{A} \pm 0,03)\end{array}$ \\
\hline 1 & 72,48 & 72,22 & 73,36 \\
2 & 72,36 & 72,45 & 73,15 \\
3 & 72,46 & 72,29 & 73,15 \\
Promedio & $72,43 \%$ & $72,32 \%$ & $73,24 \%$ \\
DS & 0,06 & 0,12 & 0,11 \\
DSR\% & 0,001 & 0,002 & 0,002 \\
\hline
\end{tabular}

\%IA: \% ingrediente activo, DS: desviación estándar, DSR\%: desviación estándar relativa
Posteriormente, se realizó la comparación con dos lotes distintos del producto que se analizaron por HPLC y GC, obteniendo los resultados incluidos en el Cuadro 4. En este se observan resultados muy exactos y precisos donde el DSR no llega al 0,5\%, y del mismo modo se observa que para la prueba de $\mathrm{F}$ realizada, el valor estadístico calculado es mayor que el valor de $\mathrm{F}$ teórico, lo cual hace referencia a que el método por HPLC es menos preciso que el método por GC. Sin embargo, se considera que los valores de ambos métodos no presentan diferencias significativas y por ende las diferencias pueden ser consideradas normales al cambiar de técnica analítica.

\section{Validación de clorotalonil en GC y HPLC}

Selectividad: Los resultados incluidos en el Cuadro 5 evidencian que las seis muestras de placebo ensayadas con ambos métodos se encuentran dentro del criterio de aceptación (3\%), lo cual indica que todos los ingredientes con que se lleva a cabo la formulación del producto de clorotalonil, no van a producir ninguna interferencia en las mediciones del ingrediente activo y ambos métodos se consideran selectivos.

Ámbito de trabajo y linealidad: seguidamente, en el método por GC el intervalo de trabajo más adecuado $\left(\mathrm{r}^{2}\right.$ mayor a 0,995) de trabajo es de 4000 a $6000 \mathrm{mg} / \mathrm{L}$, ya que en rangos distintos se afecta la linealidad y por ende los resultados de análisis del clorotalonil. Al igual, para el método por HPLC, las concentraciones para el ámbito de trabajo fueron de 60 a 140mg/L para asegurar la linealidad del método de análisis.

\section{CUADRO 4}

Resultados del método por GC contra el método HPLC en dos lotes de muestras

\begin{tabular}{|c|c|c|c|c|c|c|c|c|}
\hline \multirow[b]{2}{*}{ Muestra } & \multicolumn{4}{|c|}{ Método GC } & \multicolumn{4}{|c|}{ Método HPLC } \\
\hline & $\begin{array}{c}\text { Concentración } \\
(\% \mid A \pm 0,01)\end{array}$ & $\begin{array}{l}\text { Promedio } \\
(\% \mid A \pm 0,01)\end{array}$ & DS & DSR\% & $\begin{array}{c}\text { Concentración } \\
(\% \mid A \pm 0,03)\end{array}$ & $\begin{array}{c}\text { Promedio } \\
(\% \mid A \pm 0,03)\end{array}$ & DS & DSR\% \\
\hline L1-M1 & 72,47 & 72,40 & 0,125 & 0,17 & 72,09 & 72,30 & 0,315 & 0,44 \\
\hline L1-M2 & 72,51 & & & & 72,64 & & & \\
\hline L1-M3 & 72,26 & & & & 72,10 & & & \\
\hline L2-M1 & 72,19 & 72,20 & 0,015 & 0,02 & 71,55 & 71,47 & 0,264 & 0,37 \\
\hline L2-M2 & 72,22 & & & & 71,18 & & & \\
\hline L2-M3 & 72,20 & & & & 71,69 & & & \\
\hline \multicolumn{2}{|c|}{$\mathrm{H}_{0}: \sigma_{1}^{2}=\sigma_{1}^{2}$} & \multicolumn{3}{|c|}{ Estadístico $\mathrm{F}=10,65$} & \multicolumn{4}{|c|}{$\mathrm{F}_{\text {teórico }}=7,146$} \\
\hline
\end{tabular}


CUADRO 5

Resultados de selectividad presentados por ambos métodos

\begin{tabular}{lcc}
\multicolumn{1}{c}{ Medición } & Método GC & Método HPLC \\
\hline Placebo 1 & $0,009 \%$ & $1,35 \%$ \\
Placebo 2 & $0,008 \%$ & $0,50 \%$ \\
Placebo 3 & $0,021 \%$ & $0,48 \%$ \\
Placebo 4 & $0,011 \%$ & $0,74 \%$ \\
Placebo 5 & $0,018 \%$ & $0,82 \%$ \\
Placebo 6 & $0,014 \%$ & $0,42 \%$ \\
Cumple/ No Cumple & Cumple & Cumple \\
Criterio de aceptación $\leq 3 \%$ & & \\
\hline
\end{tabular}

Curvas A,B,C Método GC

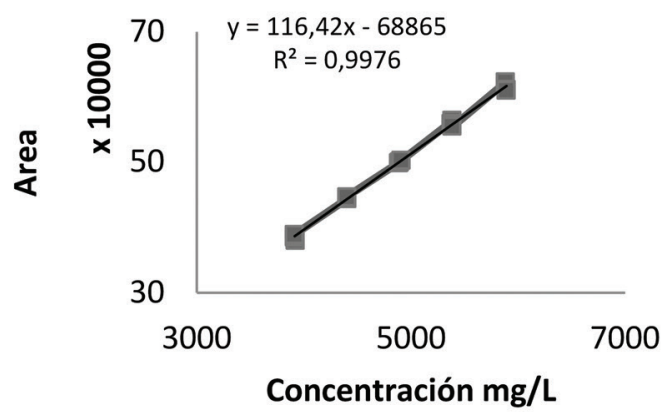

$r^{2}$ del método GC. Sin embargo, esto no es suficiente para comprobar que los métodos cumplen una buena linealidad, por esto se evalúan otros criterios, para confirmar que la linealidad es aceptable. Al determinar la homogeneidad de las varianzas para las distintas curvas (Fig. 2), la cual refleja un comportamiento homocedástico para ambos métodos.

Este criterio se basó en la prueba de análisis de Levene, en el cual se evalúan las varianzas de las tres curvas y se determina si hay similitud entre ellas. Para este caso se planteó una hipótesis nula en la cual hace referencia a que las varianzas entre las curvas son iguales. Como se

\section{Curvas A,B,C Método HPLC}

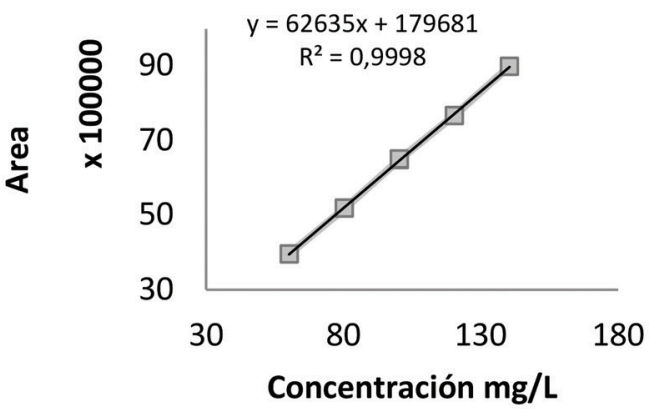

Fig. 1. Linealidad para ambos métodos (superposición de las tres curvas de calibración para cada método).

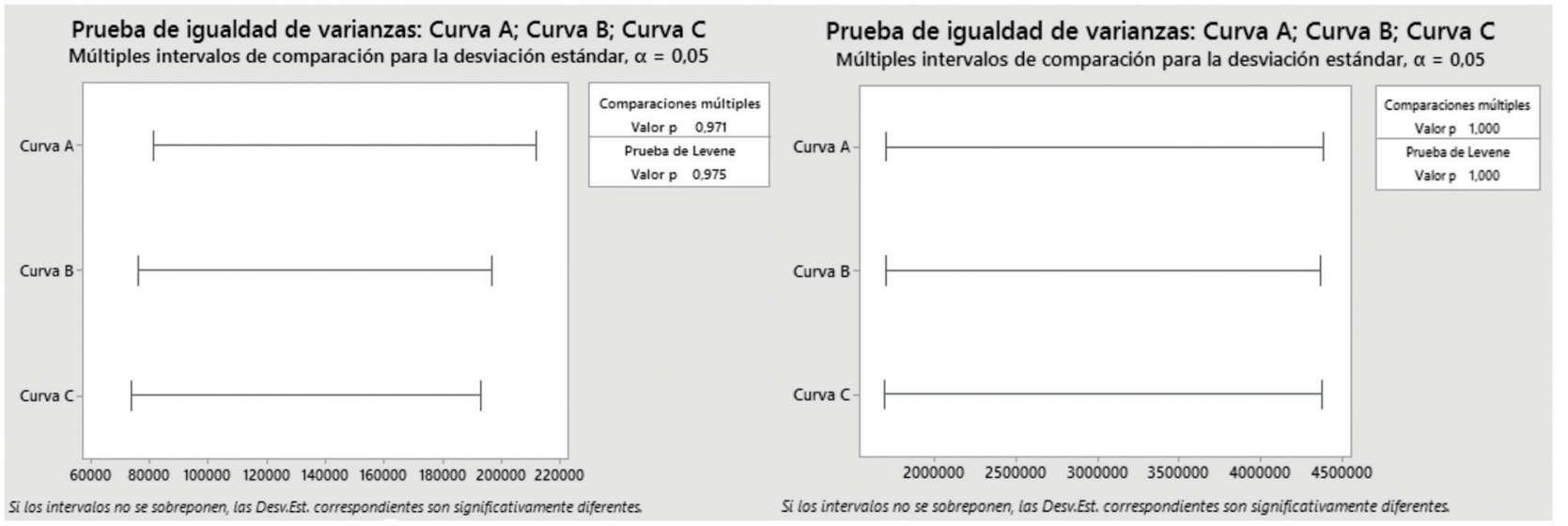

Fig. 2. Análisis de homocedasticidad por GC y por HPLC respectivamente.

Derivado del ámbito de trabajo (Fig. 1), que para los dos métodos la linealidad cumple con el criterio de aceptación, cuyos coeficientes de determinación son mayores a 0,995 lo que indica que los métodos presentan buena linealidad. De acuerdo con lo obtenido se destaca que las curvas para el método HPLC, presentan una mejor linealidad debido a que sus $r^{2}$ son más cercanos a 1 que los puede ver en la gráfica que se obtuvo para el método GC, las varianzas de las tres curvas tienden a variar un poco entre ellas, sin embargo el valor de $P$ que se obtuvo fue de 0,975 el cual es mayor a 0,05 (para un nivel de confianza del $95 \%$ ), por lo tanto no se logra rechazar la hipótesis nula y se demuestra que no hay diferencia significativa entre las varianzas de las tres curvas. 
Del mismo modo para el análisis de homocedasticidad del método HPLC, se determinó un ANOVA por el programa estadístico Minitab, cuyo resultado obtenido para el valor de $P$ fue de 1 , esto indica que también es mayor al nivel de significancia $(a=0,05)$, por lo tanto, tampoco se rechaza la hipótesis nula en este método. Se puede ver que en este método los tamaños de las varianzas para las tres curvas son consideradas iguales.

Límite de Detección y de Cuantificación: En cuanto al límite de detección y al de cuantificación (cuadro 6) se obtiene que para el método de GC lo mínimo que se puede detectar en un análisis de clorotalonil es una concentración de 593,13mg/L y en el método de HPLC es de $2,2 \mathrm{mg} / \mathrm{L}$ debido a que el HPLC es más sensible y por ende trabaja con concentraciones mucho menores al equipo de Gases. Por otro lado, está el límite de cuantificación que para el método GC se obtiene $651,65 \mathrm{mg} / \mathrm{L}$ y para el método por HPLC se obtuvo una concentración de $5,49 \mathrm{mg} / \mathrm{L}$ estas son las dos concentraciones mínimas en las cuales se puede cuantificar las muestras con precisión y exactitud aceptables.

Repetibilidad (Precisión): Con respecto a la precisión de los métodos (Cuadro 7) se puede observar que los resultados para la repetibilidad de ambos métodos cumplen con el criterio de aceptación establecido por el reglamento de validación de métodos analíticos, siendo todos menores al $2 \%$ del coeficiente de variación, lo cual indica que el grado de concordancia entre los resultados de mediciones de las muestras sucesivas es aceptable.

Precisión intermedia: En este parámetro según se muestra en el cuadro 8, en el método por GC se analizaron las mismas muestras de clorotalonil para determinar el porcentaje de ingrediente activo en dos diferentes equipos de cromatografía de gases que cuenta el laboratorio, con las mismas condiciones de análisis, solo se varió el equipo, además las lecturas se realizaron el mismo día, mismo analista y de forma simultánea. De esta forma se puede notar que en ambos equipos los resultados no presentan diferencias significativas, los dos cuentan con una buena precisión debido a que los valores no superan el criterio de aceptación establecido para este parámetro. Sin embargo, de acuerdo a la prueba $\mathrm{F}$ realizada se determina que el equipo de marca Shimadzu es más preciso que el equipo de marca Agilent.

Por otro lado, para el método por HPLC (Cuadro 9), se realizó cambio de analista, preparando por separado las

CUADRO 6

Resultados del límite de detección y cuantificación en ambos métodos

\begin{tabular}{lcc}
\multicolumn{1}{c}{ Ítem } & GC Concentración $(\mathrm{mg} / \mathrm{L})$ & HPLC Concentración $(\mathrm{mg} / \mathrm{L})$ \\
Promedio del Blanco & 592,06 & 0,79 \\
DS muestras enriquecidas & 0,36 & 0,47 \\
Límite de Detección & 593,13 & 2,2 \\
Límite de Cuantificación & 651,65 & 5,49 \\
\hline
\end{tabular}

CUADRO 7

Resultados de repetibilidad en las validaciones de ambos métodos

\begin{tabular}{ccccccc} 
Medición & $\begin{array}{c}\text { Nivel 90\% } \\
(\mathrm{mg} / \mathrm{L})\end{array}$ & $\begin{array}{c}\text { Nivel 100\% } \\
(\mathrm{mg} / \mathrm{L})\end{array}$ & $\begin{array}{c}\text { Nivel 110\% } \\
(\mathrm{mg} / \mathrm{L})\end{array}$ & $\begin{array}{c}\text { Nivel 90\% } \\
(\mathrm{mg} / \mathrm{L})\end{array}$ & $\begin{array}{c}\text { Nivel 100\% } \\
(\mathrm{mg} / \mathrm{L})\end{array}$ & $\begin{array}{c}\text { Nivel 110\% } \\
(\mathrm{mg} / \mathrm{L})\end{array}$ \\
\hline 1 & 3231,64 & 3696,57 & 4131,56 & 67,17 & 79,87 & 93,16 \\
2 & 3320,59 & 3706,17 & 4134,43 & 67,09 & 79,92 & 93,12 \\
3 & 3353,08 & 3725,46 & 4138,20 & 66,68 & 79,91 & 93,04 \\
4 & 3347,21 & 3697,09 & 4138,91 & 67,04 & 79,37 & 93,25 \\
5 & 3345,63 & 3716,56 & 4136,49 & 66,72 & 79,42 & 93,01 \\
6 & 3301,71 & 3716,33 & 4133,72 & 67,11 & 79,89 & 93,19 \\
Promedio & 3316,64 & 3709,70 & 4135,55 & 66,97 & 79,73 & 93,13 \\
DSR\% & 1,39 & 0,32 & 0,07 & 0,32 & 0,33 & 0,10 \\
Cumple/ No cumple & Cumple & Cumple & Cumple & Cumple & Cumple & Cumple \\
\hline
\end{tabular}

Criterio de aceptación: $\% C V \leq 2 \%$. 
CUADRO 8

Medición de precisión intermedia para el método GC por cambio de equipo

\begin{tabular}{|c|c|c|c|c|c|c|}
\hline \multirow[t]{2}{*}{ Medición } & \multicolumn{3}{|c|}{$\begin{array}{c}\text { GC SHIMADZU } \\
y=116,42 x-68865 \\
R^{2}=0,9976\end{array}$} & \multicolumn{3}{|c|}{$\begin{array}{c}\text { GC AGILENT } \\
\mathrm{y}=0,3955 \mathrm{x}+23,157 \\
\mathrm{R}^{2}=0,9989\end{array}$} \\
\hline & $\begin{array}{l}\text { Muestra } 1 \\
(\% \mid A \pm 0,1)\end{array}$ & $\begin{array}{l}\text { Muestra } 2 \\
(\% \mid A \pm 0,1)\end{array}$ & $\begin{array}{l}\text { Muestra } 3 \\
(\% \mid A \pm 0,1)\end{array}$ & $\begin{array}{l}\text { Muestra } 1 \\
(\% \mid A \pm 0,3)\end{array}$ & $\begin{array}{l}\text { Muestra } 2 \\
(\% \mid A \pm 0,3)\end{array}$ & $\begin{array}{l}\text { Muestra } 3 \\
(\% \mid A \pm 0,3)\end{array}$ \\
\hline 1 & 72,63 & 71,05 & 72,37 & 70,81 & 71,55 & 73,07 \\
\hline 2 & 72,47 & 71,13 & 72,27 & 72,09 & 71,18 & 72,93 \\
\hline 3 & 72,09 & 71,03 & 72,17 & 72,64 & 71,69 & 73,09 \\
\hline Promedio & \multicolumn{3}{|c|}{71,91} & \multicolumn{3}{|c|}{72,12} \\
\hline DS & \multicolumn{3}{|c|}{0,65} & \multicolumn{3}{|c|}{0,86} \\
\hline DSR\% & \multicolumn{3}{|c|}{0,91} & \multicolumn{3}{|c|}{1,19} \\
\hline Cumple/ No cumple & \multicolumn{3}{|c|}{ Cumple } & \multicolumn{3}{|c|}{ Cumple } \\
\hline $\mathrm{H}_{0}: \sigma^{2}{ }_{1}=\sigma^{2}{ }_{1}$ & \multicolumn{3}{|c|}{ Estadístico $\mathrm{F}=9,43$} & \multicolumn{3}{|c|}{$\mathrm{F}_{\text {teórico }}=4,43$} \\
\hline
\end{tabular}

Criterio de aceptación: \%CV $\leq 2 \%$

CUADRO 9

Medición de precisión intermediaria para el método HPLC por cambio de analista

\begin{tabular}{|c|c|c|c|c|c|c|}
\hline \multirow[t]{2}{*}{ Medición } & \multicolumn{3}{|c|}{$\begin{array}{c}\text { Analista } 1 \\
y=116,42 x-68865 \\
R^{2}=0,9976\end{array}$} & \multicolumn{3}{|c|}{$\begin{array}{c}\text { Analista } 2 \\
y=0,3955 x+23,157 \\
R^{2}=0,9989\end{array}$} \\
\hline & $\begin{array}{l}\text { Muestra } 1 \\
(\% \mid A \pm 0,1)\end{array}$ & $\begin{array}{l}\text { Muestra } 2 \\
(\% \mid A \pm 0,1)\end{array}$ & $\begin{array}{l}\text { Muestra } 3 \\
(\% \mid A \pm 0,1)\end{array}$ & $\begin{array}{l}\text { Muestra } 1 \\
(\% \mid A \pm 0,3)\end{array}$ & $\begin{array}{c}\text { Muestra } 2 \\
(\% \mid A \pm 0,3)\end{array}$ & $\begin{array}{l}\text { Muestra } 3 \\
(\% \mid A \pm 0,3)\end{array}$ \\
\hline 1 & 72,36 & 72,67 & 73,12 & 72,40 & 72,80 & 73,01 \\
\hline 2 & 72,90 & 72,28 & 72,98 & 72,53 & 72,91 & 73.06 \\
\hline 3 & 72,45 & 72,39 & 73,04 & 72,34 & 71,75 & 73,11 \\
\hline Promedio & \multicolumn{3}{|c|}{72,69} & \multicolumn{3}{|c|}{72,61} \\
\hline DS & \multicolumn{3}{|c|}{0,33} & \multicolumn{3}{|c|}{0,45} \\
\hline DSR\% & \multicolumn{3}{|c|}{0,45} & \multicolumn{3}{|c|}{0,62} \\
\hline Cumple/ No cumple & \multicolumn{3}{|c|}{ Cumple } & \multicolumn{3}{|c|}{ Cumple } \\
\hline \multicolumn{7}{|c|}{ Criterio de aceptación: \%CV $\leq 2 \%$} \\
\hline \multicolumn{2}{|l|}{$\mathrm{H}_{0}: \sigma^{2}{ }_{1}=\sigma^{2}{ }_{1}$} & \multicolumn{2}{|c|}{ Estadístico F=7,367 } & \multicolumn{3}{|c|}{$\mathrm{F}_{\text {teórico }}=4,433$} \\
\hline
\end{tabular}

mismas muestras. Se puede observar que los resultados para ambos analistas cumplen con el criterio de aceptación, siendo menores al $2 \%$ de coeficiente de variación y del mismo modo se puede determinar que la variación entre los resultados de los analistas no es significativa, sin embargo el analista dos es más preciso que el analista uno de acuerdo a la prueba $\mathrm{F}$ realizada. Por lo tanto, se puede considerar que la precisión y la reproducibilidad para ambos métodos son aceptables.

Robustez: Esta prueba se realizó a ocho muestras de clorotalonil analizadas en dos momentos distintos, donde el día uno equivale al primer día en que se prepararon las muestras, y el día tres equivale a tres días posteriores a la preparación de las muestras. Esto se hizo con el fin de medir las variaciones que presentan las muestras en los resultados de sus análisis con respecto al tiempo, temperatura, luz y todos los factores ambientales que puedan presentarse dentro del laboratorio. Como se puede observar (Cuadro 10) todas las muestras tienden a presentar un comportamiento similar, en el cual su nivel de concentración es más bajo en su segunda medición comparándolos con los resultados de la primera medición, lo cual indica que las muestras preparadas se van descomponiendo durante el transcurso del tiempo. El método HPLC es el que presenta una mayor variedad, sin embargo, se encuentra dentro del criterio de aceptación, el cual debe ser menor a 3\%; y el método GC también cumple con este criterio. 
CUADRO 10

Resultados de la prueba de robustez para la validación de los dos métodos

\begin{tabular}{|c|c|c|c|c|}
\hline \multirow{3}{*}{ Muestras } & \multicolumn{4}{|c|}{ Porcentajes Ingrediente Activo } \\
\hline & \multicolumn{2}{|c|}{ Método GC } & \multicolumn{2}{|c|}{ Método HPLC } \\
\hline & Día $1(\% \mid A \pm 0,1)$ & Día $3(\% \mid A \pm 0,1)$ & Día $1(\% \mid A \pm 0,3)$ & Día $3(\% \mid A \pm 0,3)$ \\
\hline 1 & 72,85 & 71,92 & 72,11 & 70,59 \\
\hline 2 & 72,47 & 71,56 & 72,06 & 69,98 \\
\hline 3 & 72,03 & 71,02 & 72,44 & 70,65 \\
\hline 4 & 72,26 & 71,27 & 72,57 & 70,91 \\
\hline 5 & 72,12 & 71,19 & 72,24 & 70,66 \\
\hline 6 & 71,73 & 70,90 & 72,32 & 70,26 \\
\hline 7 & 72,06 & 71,18 & 72,28 & 70,47 \\
\hline 8 & 73,02 & 72,06 & 72,83 & 70,98 \\
\hline \% Variación & \multicolumn{2}{|c|}{$1,28 \%$} & \multicolumn{2}{|c|}{$2,48 \%$} \\
\hline Cumple/No cumple & \multicolumn{2}{|c|}{ Cumple } & \multicolumn{2}{|c|}{ Cumple } \\
\hline
\end{tabular}

Criterio de aceptación: el porcentaje de Variación debe ser $\leq 3 \%$.

CUADRO 11

Resultados de veracidad por GC

\begin{tabular}{ccccc} 
Nivel & \% Enriquecido & Contenido teórico $(\mathrm{mg} / \mathrm{L})$ & Contenido medido $(\mathrm{mg} / \mathrm{L})$ & $\%$ Recuperación \\
1 & 90,61 & 3262 & 3301,8 & 101,2 \\
2 & 100,18 & 3606 & 3709,4 & 102,8 \\
3 & 110,73 & 4015 & 4134,7 & 102,9 \\
4 & 121,84 & 4418 & 4538,4 & 102,7 \\
5 & 131,49 & 4786 & 4854.9 & 101,4 \\
\hline
\end{tabular}

Criterio de aceptación: \%Recuperación $\geq 97 \%$ y $\leq 103 \%$.

CUADRO 12

Resultados de veracidad por HPLC

\begin{tabular}{ccccc} 
Nivel & \% Enriquecido & Contenido teórico $(\mathrm{mg} / \mathrm{L})$ & Contenido medido $(\mathrm{mg} / \mathrm{L})$ & \% Recuperación \\
1 & 93,13 & 67,06 & 66,98 & 99,89 \\
2 & 113.27 & 81,56 & 79,89 & 97,96 \\
3 & 129,85 & 93,49 & 93,11 & 99,59 \\
4 & 145,38 & 104,66 & 103,26 & 98,66 \\
5 & 160,44 & 115,52 & 112,74 & 97,59 \\
\hline
\end{tabular}

Criterio de aceptación: \%Recuperación $\geq 97 \%$ y $\leq 103 \%$.

Veracidad: De acuerdo con los datos obtenidos para la veracidad tanto del método GC (Cuadro 11) como del método HPLC (Cuadro 12), se logra determinar que la muestra en ambos métodos de comporta de forma muy similar, sin importar los niveles a los que se prepara la muestra los resultados son muy acordes a los resultaos esperados, por lo que todos los niveles se encuentran dentro del rango de criterio de aceptación el cual es $97-103 \%$, lo cual da señal que los métodos presentan una buena exactitud. Sin embargo, también se puede notar que para el método GC, los niveles de recuperación son mayores a 100, lo cual indica que los valores obtenidos tienden a ser mayores a los esperados y en el método HPLC los valores obtenidos suelen ser menores a los esperados (Ministerio de Industria, Turismo y Comercio, 2018) pero siguen estando dentro del rango aceptado. 
Del mismo modo, de acuerdo con la correlación entre el contenido medido y el porcentaje enriquecido, las muestras presentan un buen comportamiento lineal, ya que en ambos métodos el $\mathrm{R}^{2}$ de la curva son mayores a 0,995 que es el valor mínimo aceptado.

Sesgo: al igual que en la veracidad, se determinó el porcentaje de recuperación de las muestras, en comparación con una muestra estándar con concentración determinada (72,205\% de clorotalonil). Se puede notar en el cuadro 13, que ambos métodos cumplen con el criterio de aceptación, debido a que todos los valores obtenidos están dentro del rango $97-103 \%$, y la diferencia relativa con respecto al valor verdadero es mucho menor al $10 \%$ en todos los casos. Sin embargo, se puede ver que también en esta prueba el método GC tiene un comportamiento que conlleva a resultados ligeramente más altos a los esperados y el método HPLC a valores más bajos que los esperados.
Comparación de métodos: Para este caso se tienen dos medias muestrales, tomando como hipótesis nula que los dos métodos proporcionan el mismo resultado, es decir $\mathrm{H}_{0}: \mu_{1}=\mu_{2}$, se necesita probar si $\left(\mathrm{X}_{1}-\mathrm{X}_{2}\right)$ difiere significativamente de cero. Tenemos 10 grados de libertad, por lo tanto el valor crítico para un valor de confianza de $95 \%$ es de $t_{8}=2,22$. Puesto que el valor experimental de $t$ $(0,823)$ es menor que el crítico, quiere decir que no se rechaza la hipótesis nula, por lo tanto la diferencia entre los resultados de los dos métodos (Cuadro 14) no difieren significativamente entre sí. (Miller \& Miller, 2002).

En cuanto al costo del análisis, el solvente utilizado en el método GC (Acetona) es mucho más barato que el utilizado en el método HPLC (Acetonitrilo), los tiempos de preparación de las muestras son muy parecidos en ambos métodos, y en cuanto al tiempo de análisis en el equipo, los dos duran alrededor de 5 minutos cada muestra.

CUADRO 13

Resultados de exactitud en ambos métodos

\begin{tabular}{|c|c|c|c|c|c|c|}
\hline \multirow[b]{2}{*}{ Réplica } & \multicolumn{3}{|c|}{ GC } & \multicolumn{3}{|c|}{ HPLC } \\
\hline & $\begin{array}{l}\text { Muestra } 1 \\
(\% \mid A \pm 0,1)\end{array}$ & $\begin{array}{l}\text { Muestra } 2 \\
(\% \mid A \pm 0,1)\end{array}$ & $\begin{array}{l}\text { Muestra } 3 \\
(\% \mid A \pm 0,1)\end{array}$ & $\begin{array}{l}\text { Muestra } 1 \\
(\% \mid A \pm 0,3)\end{array}$ & $\begin{array}{l}\text { Muestra } 2 \\
(\% \mid A \pm 0,3)\end{array}$ & $\begin{array}{l}\text { Muestra } 3 \\
(\% \mid A \pm 0,3)\end{array}$ \\
\hline 1 & 72,18 & 72,61 & 73,41 & 71,75 & 70,14 & 71,37 \\
\hline 2 & 72,11 & 72,80 & 73,46 & 71,66 & 70,18 & 71,34 \\
\hline 3 & 72,82 & 73,18 & 73,53 & 71,23 & 70,17 & 71,28 \\
\hline Promedio & 72,37 & 72,86 & 73,47 & 71,54 & 70,16 & 71,33 \\
\hline Cn Teórica & 72,205 & 72,205 & 72,205 & 72,205 & 72,205 & 72,205 \\
\hline Sesgo ( $\leq 10 \%)$ & 0,23 & 0,91 & 1,75 & 0,92 & 2,83 & 1,21 \\
\hline \%Recuperación & 100,23 & 100,91 & 101,75 & 99,08 & 97,2 & 98,79 \\
\hline Cumple/ No cumple & Cumple & Cumple & Cumple & Cumple & Cumple & Cumple \\
\hline
\end{tabular}

Criterio de Aceptación 97-103\%

CUADRO 14

Comparación de métodos GC vrs HPLC con muestras reales de clorotalonil

\begin{tabular}{ccc} 
Muestra & GC & HPLC \\
\hline 1 & Concentración \%IA $\pm 0,1$ & Concentración \%lA $\pm 0,3$ \\
2 & 72,37 & 72,09 \\
3 & 72,71 & 72,64 \\
4 & 72,26 & 72,10 \\
5 & 72,69 & 72,55 \\
6 & 72,22 & 72,18 \\
Promedio & 72,75 & 72,69 \\
DS & 72,5 & 72,38 \\
DSR\% & 0,24 & 0,28 \\
\hline
\end{tabular}




\section{AGRADECIMIENTOS}

Agradecemos al personal del Laboratorio de Control de Calidad de FORMUQUISA, por la apertura para realizar trabajos como este, y por todo el apoyo brindado durante el proceso de indagación.

\section{REFERENCIAS}

Alatorre, R. (1999). Manejo integrado de plagas. México: Secretaria de Agricultura, Ganadería, Desarrollo Rural Pesca y Alimentación.

Álvarez, A., Támbara, Y., Álvarez, F., Besada, V., \& González, L. (2014). Calificación de un cromatógrafo de gases Agilent Technologies 7890A utilizado en la industria biotecnológica. Centro de Ingeniería Genética y Biotecnología (CIGB), La Habana, Cuba. Revista Cubana de Farmacia, 48(2), 350-358. Recuperado de http://scielo.sld.cu/pdf/ far/v48n3/far02314.pdf

Álvarez, M., Conci, M., \& Peccoud, C. (2009). Pocos ganan, muchos pierden. Soja, agroquímicos y salud. Departamento Rio II - Córdoba, Argentina: Editorial EDUVIM.

AOAC (2016). Official Methods of Analysis of AOAC International 20th edition. MD, EEUU: Gaithersburg.

Ascuasati, A. (2012). Plagas Domésticas: Historia Patologías Plaguicidas Control. Bloomington, IN, EEUU: Editorial Palibrio.

Decreto No 24337-MAG-S. (1995). Reglamento sobre registro, uso y control de plaguicidas agrícolas y coadyuvantes. Recuperado de http://canapep.com/ wp-content/uploads/2015/12/Decreto-24337-MAG-Sde-Reglamento-de-registro-y-uso-de-plaguicidas-ycoadyuvantes.pdf [Consulta 17 nov. 2016].

Duran, D. (2011). Análisis fisicoquímicos de productos farmacéuticos en las diferentes etapas del proceso de la industria farmacéutica. Universidad de Carabobo, Venezuela. Recuperado de http://portal.facyt.uc.edu.ve/pasantias/ informes/P75515319.pdf

EcoTenda. (2013). Manual de insecticidas, fungicidas y fitofortificantes ecológicos. Agricultura y jardinería ecológica. Recuperado de http://caminosostenible.org/wp-content/uploads/BIBLIOTECA/manual_insecticidas.pdf

Ente Costarricense de Acreditación. (2015). Guía para la Validación de Métodos. Versión 3. San José, Costa Rica. Recuperado de http://eca.or.cr/

Ferreira, P. (1996). Recursos Naturales y Sostenibilidad Agrícola en Mesoamérica. Turrialba, Costa Rica: Catie.

García, S., \& Pérez, R. (2012). Aplicaciones de la Cromatografía Líquida con Detector de Diodos y Fluorescencia al Análisis de Contaminantes Medioambientales. Centro de Investigaciones Energéticas, Medioambientales y Tecnológicas, España. Recuperado de https:// inis.iaea.org/collection/NCLCollectionStore/ Public/43/028/43028576.pdf

Gepp, V., \& Mondino, P. (2018). Apuntes sobre fungicidas. Universidad de la Republica. Recuperado de http:// www.pv.fagro.edu.uy/cursos/pvh/DocsPVH/Apuntes_ Fungicidas.pdf

Mendizábal, A. (2011). Evaluación de la degradación de Fungicidas en camas Biológicas. Universidad del Valle de Guatemala, Guatemala. Recuperado de http://glifos. concyt.gob.gt/digital/fodecyt/fodecyt\%202006.22.pdf

Menéndez, F. (2009). Higiene industrial. España: Editorial Lex Nova.

Miller, J., \& Miller, J. (2002). Estadística y Quimiometría para química analítica. Madrid, España: Pearson Prentice Hall.

Ministerio de Industria, Turismo y Comercio. (2018). Procedimiento QU-004 para la calibración equipos de cromatografía de líquidos de alta resolución (HPLC). Recuperado de https://www.cem.es/sites/default/files/ qu-004_digital.pdf

Ministerio de Salud. (2007). La problemática de los agroquímicos y sus envases, su incidencia en la salud de los trabajadores, la población expuesta y el ambiente. - 1a ed. Buenos Aires, Argentina.

Miranda, A., \& Martín, O. (2018). Cromatografía líquida (HPLC). Recuperado de http://www.ucm.es/data/cont/ docs/650-2013-12-02-gases\%201\%C3\%ADquidos.pdf

Norma RTCR-171. (1991). Plaguicidas y Coadyuvantes. Tolerancias Permitidas para la Concentración del Ingrediente Activo. Recuperado de https://www.sfe. go.cr/Decretos/Decreto\%20N\%C2\%B0\%2027035\%20 $\% 20$ Plaguicidas $\% 20 y \% 20$ Coadyuvantes. $\% 20$ Tolerancias\%20Permitidas\%20para\%20la\%20 Concentraci\%C3\%B3n\%20del\%20Ingrediente\%20Activo.pdf

Pérez, W., \& Forbes, G. (2017). División de manejo integrado de cultivos. Recuperado de http://cipotato.org/wp-content/uploads/2014/09/003862.pdf

Reyes, J., Vásquez, R., \& González, T. (2010). Introducción a la agroquímica. Puebla, México: Editorial BUAP.

Sigma-Aldrich. (2016). Intralab Validation of the EN 15662 Method for the Determination of Pesticide Residues Using a Fused-Core ${ }^{\mathrm{TM}}$ Ascentis ${ }^{\circledR}$ Express RP-Amide HPLC Column by LC-MS/MS and Clean-up by Dispersive SPE (QuEChERS). Recuperado de http://www.sigmaaldrich. com/us-export.html

Varés, L. (2006). Resistencia de los patógenos a los fungicidas. Universidad Politécnica de Madrid, España.

Villegas, A. (1993). Manejo seguro de plaguicidas: Manual para técnicos y aplicadores. Centro Internacional de Agricultura Tropical, Cali. Colombia. 